\title{
Global Synchronization for Delayed Complex Networks with Randomly Occurred Nonlinearities and Multiple Stochastic Disturbances
}

\author{
Yao $\mathrm{Wang}^{a}$, Zidong $\mathrm{Wang}^{a, b, *}$ and Jinling $\operatorname{Liang}^{c}$
}

\begin{abstract}
This paper is concerned with the synchronization problem for a new class of continuous-time delayed complex networks with stochastic nonlinearities (randomly occurred nonlinearities), interval time-varying delays, unbounded distributed delays as well as multiple stochastic disturbances. The stochastic nonlinearities and multiple stochastic disturbances are investigated here in order to reflect more realistic dynamical behaviors of the complex networks that are affected by the noisy environment. By utilizing a new matrix functional with the idea of partitioning the lower bound $\hbar_{1}$ of the time-varying delay, we employ the stochastic analysis techniques and the properties of Kronecker product to establish delay-dependent synchronization criteria that ensure the globally asymptotically mean-square synchronization of the addressed stochastic delayed complex networks. The sufficient conditions obtained are in form of linear matrix inequalities (LMIs) whose solution can be readily solved by using the standard numerical software. A numerical example is exploited to show the applicability of the proposed results.
\end{abstract}

\section{Keywords}

Global synchronization; stochastic complex networks; stochastic nonlinearity; linear matrix inequality (LMI).

\section{INTRODUCTION}

Over the past few decades, the studies of complex networks have been an active field of research in many scientific and technical disciplines $[1,5,6,14-16,26,31-33,38]$. Many systems in nature can be described by complex networks with examples including genetic networks, information networks, the Internet, and social networks etc. Among many literature on analyzing complex networks, one of the most widely investigated dynamical behaviors of complex networks is the synchronization motion of its dynamical elements [6,14,18,33,38].

In practice, time delays are considered as ubiquitous in networks due to the fact that the information transmission within complex networks is in general not instantaneous since the signals traveling speed is limited, and it is known that the time delays may cause undesirable dynamic network behaviors such as oscillation and instability. In addition, it is noted that continuously distributed delays, either bounded or unbounded $[3,19,20,22,28]$, have received more attention since the complex network usually has a spatial nature due to the presence of parallel pathways with a variety of axon sizes and lengths. Recently, there has been a growing number of papers dealing with both discrete and distributed time-delays, see e.g. $[3,8,19,20,28]$ and the references therein.

In real-time systems, the signal transmission is usually a noisy process brought on by random fluctuations from probabilistic causes and, therefore, stochastic modeling has been of vital importance in many branches of science such as neurotransmitters and packet dropouts. It is often the case that the dynamical behaviors of complex networks are largely affected by the stochastic disturbances. Subsequently, the synchronization problem for stochastic networks has begun to receive some initial research interests. In $[16,17,29,33]$, the synchronization problems have been intensively investigated for delayed complex networks with various kinds of stochastic disturbances, where the criteria ensuring the synchronization among networks have been derived mainly based on the Lyapunov approach that is capable of coping with the different type of time-delays.

In addition to the network-induced delay and external stochastic disturbances, "random" phenomena often appear due to connections over communication channels, such as random communication delay, random measurements and random packet losses, which have recently attracted much attention in the networked control society. However, another

This work was supported in part by the Engineering and Physical Sciences Research Council (EPSRC) of the U.K. under Grant GR/S27658/01, an International Joint Project sponsored by the Royal Society of the U.K., the National Natural Science Foundation of China under Grant 60804028, the Specialized Research Fund for the Doctoral Program of Higher Education for New Teachers under Grant 200802861044, the Teaching and Research Fund for Excellent Young Teachers at Southeast University of China, and the Alexander von Humboldt Foundation of Germany.

${ }^{a}$ School of Information Sciences and Technology, Donghua University, Shanghai 200051, China.

${ }^{b}$ Department of Information Systems and Computing, Brunel University, Uxbridge, Middlesex, UB8 3PH, United Kingdom.

${ }^{c}$ Department of Mathematics, Southeast University, Nanjing 210096, China.

${ }^{*}$ Corresponding author. Email: Zidong.Wang@brunel.ac.uk. 
interesting random phenomenon, random nonlinearity, has been largely overlooked. As is well known, a wide class of practical systems are influenced by additive nonlinear disturbances that are caused by environmental circumstances. For complex networks with communication constraints, such nonlinear disturbances themselves may be subject to random abrupt changes, which may result from abrupt phenomena such as random failures and repairs of the components, changes in the interconnections of subsystems, sudden environment changes, modification of the operating point of a linearized model of a nonlinear systems, etc. In other words, the nonlinear disturbances may occur in a probabilistic way and are randomly changeable in terms of their types and/or intensity. The randomly occurred nonlinearities, also called stochastic nonlinearities, have recently received some interest in the literature. For example, in [35,37], the filtering and control problems for discrete-time systems with stochastic nonlinearities have been thoroughly investigated. Unfortunately, to the best of the authors' knowledge, the synchronization problem for complex networks experiencing randomly occurred nonlinearities with or without time-delays and stochastic disturbances has received very little attention, and the purpose of this paper is therefore to fill in such a gap.

Motivated by the above observations, in this paper, we aim to deal with the synchronization problem for stochastic delayed complex networks (SDCN) with stochastic nonlinearities, multiple stochastic disturbances, time-varying delays and continuously distributed delays. We are interested in deriving sufficient conditions for the addressed problem by employing the properties of Kronecker product [13] and the stochastic analysis techniques [2,11,24], combined with the 'delay fractioning' approach $[25,26,33]$. A novel matrix functional is constructed to attain new synchronization criteria, which are formulated in the form of linear matrix inequalities (LMIs) [4]. Note that the LMIs can be solved by using the standard numerical software.

The remainder of this paper is organized as follows. In Section II, a stochastic complex network model with mixed time-delays, stochastic nonlinearities and multiple stochastic disturbances is proposed, and some preliminaries are briefly outlined. In Section III, by utilizing the approach of 'delay fractioning' and the matrix functional method, we conduct the stochastic analysis to obtain delay-dependent sufficient criteria in terms of LMIs so as to ensure that the considered SDCN with multiple stochastic disturbances and stochastic nonlinearities are globally synchronized in the mean square. In Section IV, a numerical example is provided to show the applicability of the obtained results. The conclusions are finally drawn in Section V.

Notations: Throughout this paper, $\mathbb{R}^{n}$ and $\mathbb{R}^{n \times m}$ denote, respectively, the $n$ dimensional Euclidean space and the set of all $n \times m$ real matrices. $P>0$ means that matrix $P$ is real, symmetric and positive definite. $I$ and 0 denote the identity matrix and the zero matrix with compatible dimensions, respectively; and $\operatorname{diag}\{\cdots\}$ stands for a block-diagonal matrix, $\operatorname{col}\{\cdots\}$ denotes a matrix column with blocks given by the matrices in $\{\cdots\}$. If $A$ is a matrix, the notation $\lambda_{\max }(A)$ means the largest eigenvalue of $A$. The superscript " $T$ " stands for matrix transposition and the asterisk " $*$ " in a matrix is used to represent the term which is induced by symmetry. The Kronecker product of matrices $Q \in \mathbb{R}^{m \times n}$ and $R \in \mathbb{R}^{p \times q}$ is a matrix in $\mathbb{R}^{m p \times n q}$ and denoted as $Q \otimes R$. We let $\mathcal{C}\left((-\infty, 0] ; \mathbb{R}^{n}\right)$ denote the family of continuous functions $\varphi$ from $(-\infty, 0]$ to $\mathbb{R}^{n}$ with the norm $|\varphi|=\sup _{-\infty \leq \theta \leq 0}\|\varphi(\theta)\|$, where $\|\cdot\|$ is the Euclidean norm on $\mathbb{R}^{n}$. Moreover, let $\left(\Omega, \mathcal{F},\left\{\mathcal{F}_{t}\right\}_{t \geq 0}, P\right)$ be a complete probability space with a filtration $\left\{\mathcal{F}_{t}\right\}_{t \geq 0}$ satisfying the usual conditions (i.e., the filtration contains all $P$-null sets and is right continuous). Denote by $L_{\mathcal{F}_{0}}^{p}\left((-\infty, 0] ; \mathbb{R}^{n}\right)$ the family of all $\mathcal{F}_{0}$-measurable $\mathcal{C}\left((-\infty, 0] ; \mathbb{R}^{n}\right)$-valued random variables $\xi=\{\xi(\theta):-\infty<\theta \leq 0\}$ such that $\sup _{-\infty<\theta \leq 0} \mathbb{E}\left\{|\xi(\theta)|^{p}\right\}<\infty$, where $\mathbb{E}\{\cdot\}$ stands for the mathematical expectation operator with respect to the given probability measure $P$. Sometimes, the arguments of a function will be omitted in the analysis when no confusion arises.

\section{Problem Formulation AND PRELiminaries}

Consider the following array of delayed complex networks with multiple stochastic disturbances and stochastic nonlinearities:

$$
\begin{aligned}
d x_{i}(t)= & {\left[A x_{i}(t)+D x_{i}(t-\tau(t))+\delta(t) B_{1} f\left(x_{i}(t)\right)+(1-\delta(t)) B_{2} g\left(x_{i}(t)\right)\right.} \\
& \left.+C \int_{-\infty}^{t} \kappa(t-s) h\left(x_{i}(s)\right) d s\right] d t+\sum_{j=1}^{N} w_{i j}^{(1)} \Gamma_{1} x_{j}(t)\left(d t+d \omega_{1}(t)\right) \\
& +\sum_{j=1}^{N} w_{i j}^{(2)} \Gamma_{2} x_{j}(t-\tau(t))\left(d t+d \omega_{2}(t)\right)+\sigma_{i}\left(t, x_{i}(t), x_{i}(t-\tau(t))\right) d \omega_{3}(t), \quad i=1,2, \ldots, N,
\end{aligned}
$$

where $x_{i}(t)=\operatorname{col}\left\{x_{i 1}(t), \ldots, x_{i n}(t)\right\} \in \mathbb{R}^{n}$ is the state vector of the $i$ th network at time $t ; A, D, B_{1}$ and $B_{2}$ are the known real constant matrices; $\Gamma_{1}, \Gamma_{2} \in \mathbb{R}^{n \times n}$ represent the inner-coupling between the subsystems at time $t$ and $t-\tau(t)$, 
respectively; $W^{(1)}=\left(w_{i j}^{(1)}\right)_{N \times N}$ and $W^{(2)}=\left(w_{i j}^{(2)}\right)_{N \times N}$ are the outer-coupling configuration matrices representing the coupling strength and the topological structure of the complex networks; furthermore, $\sigma_{i}(\cdot, \cdot, \cdot): \mathbb{R} \times \mathbb{R}^{n} \times \mathbb{R}^{n} \rightarrow \mathbb{R}^{n}$ is the noise intensity function vector, and $\omega_{k}(t)(k=1,2,3)$ are mutually independent scalar Brownian motions defined on $(\Omega, \mathcal{F}, \mathcal{P})$ satisfying

$$
\mathbb{E}\left\{\omega_{k}(t)\right\}=0 \text { and } \mathbb{E}\left\{\left[\omega_{k}(t)\right]^{2}\right\}=d t .
$$

Moreover, $\kappa(\cdot):[0,+\infty) \rightarrow[0,+\infty)$ is the distributed time-delay kernel; and the discrete time delay $\tau(t)$ is a time-varying differentiable function satisfying [9]:

$$
0<\hbar_{1} \leq \tau(t) \leq \hbar_{2}, \quad \dot{\tau}(t) \leq \mu,
$$

where $\hbar_{1}, \hbar_{2}$ and $\mu$ are constants. Finally, $f\left(x_{i}(t)\right)=\operatorname{col}\left\{f_{1}\left(x_{i 1}(t)\right), f_{2}\left(x_{i 2}(t)\right), \ldots, f_{n}\left(x_{i n}(t)\right)\right\}, g\left(x_{i}(t)\right)=\operatorname{col}\left\{g_{1}\left(x_{i 1}(t)\right)\right.$, $\left.g_{2}\left(x_{i 2}(t)\right), \ldots, g_{n}\left(x_{i n}(t)\right)\right\}$ and $h\left(x_{i}(t)\right)=\operatorname{col}\left\{h_{1}\left(x_{i 1}(t)\right), h_{2}\left(x_{i 2}(t)\right), \ldots, h_{n}\left(x_{i n}(t)\right)\right\}$ are continuous nonlinear functions, and $\delta(t)$ is a stochastic variable that describes the following random events for the system (1):

$$
\left\{\begin{array}{l}
\text { Event } 1: \text { The system }(1) \text { experiences nonlinear function } f(\cdot), \\
\text { Event } 2: \text { The system (1) experiences nonlinear function } g(\cdot) \text {. }
\end{array}\right.
$$

Letting $\delta(t)$ be a Bernoulli distributed sequence defined by

$$
\delta(t)= \begin{cases}1: & \text { if Event } 1 \text { occurs } \\ 0: & \text { if Event } 2 \text { occurs }\end{cases}
$$

it follows that $\delta(t)$ satisfies

$$
\operatorname{Prob}\{\delta(t)=1\}=\mathbb{E}\{\delta(t)\}=\delta_{0}, \quad \operatorname{Prob}\{\delta(t)=0\}=1-\mathbb{E}\{\delta(t)\}=1-\delta_{0},
$$

where the constant $\delta_{0} \in[0,1]$ reflects the occurrence probability of the event of the nonlinear functions $f(\cdot)$ and $g(\cdot)$. It is further assumed that the variables $\delta(t)$ and $\omega_{k}(t)(k=1,2,3)$ are mutually independent.

Remark 1: In this paper, the random variable $\delta(t)$ is used to model the probability distribution of the nonlinear functions. To our knowledge, this represents the first attempt to take into account the occurrence of different nonlinear functions in a probabilistic way for the addressed complex networks. In other words, in the complex network (1), the two terms $\delta(t) B_{1} f\left(x_{i}(t)\right)$ and $(1-\delta(t)) B_{2} g\left(x_{i}(t)\right)$ can be used to account for the binary switch between these two nonlinear functions according to the given probability distribution.

Remark 2: It follows from the given hypothesis that $\mathbb{E}\left\{\delta(t)-\delta_{0}\right\}=0$ and $\mathbb{E}\left\{\left(\delta(t)-\delta_{0}\right)^{2}\right\}=\delta_{0}\left(1-\delta_{0}\right)$. As pointed out in [39], $\delta(t)$ is a Markovian process and, for the following use, it is assumed that $\delta(t)$ follows an unknown but exponential distribution of switchings.

Throughout this paper, the following assumptions are needed:

Assumption 1: [6] The outer-coupling configuration matrices of the complex networks (1) satisfy

$$
w_{i j}^{(q)}=w_{j i}^{(q)} \geq 0 \quad(i \neq j), \quad w_{i i}^{(q)}=-\sum_{j=1, j \neq i}^{N} w_{i j}^{(q)} \quad(q=1,2 ; i, j=1,2, \ldots, N) .
$$

Assumption 2: [10] For $\forall u, v \in \mathbb{R}^{n}$, the nonlinear functions $f(\cdot), g(\cdot), h(\cdot)$ satisfy the following sector-bounded conditions

$$
\begin{aligned}
& {\left[f(u)-f(v)-X_{1}(u-v)\right]^{T}\left[f(u)-f(v)-X_{2}(u-v)\right] \leq 0,} \\
& {\left[g(u)-g(v)-Y_{1}(u-v)\right]^{T}\left[g(u)-g(v)-Y_{2}(u-v)\right] \leq 0,} \\
& {\left[h(u)-h(v)-H_{1}(u-v)\right]^{T}\left[h(u)-h(v)-H_{2}(u-v)\right] \leq 0,}
\end{aligned}
$$

where $X_{1}, Y_{1}, H_{1}$ and $X_{2}, Y_{2}, H_{2}$ are real constant matrices with $X_{2}-X_{1} \geq 0, Y_{2}-Y_{1} \geq 0$ and $H_{2}-H_{1} \geq 0$.

Remark 3: The nonlinear functions $f(\cdot), g(\cdot)$ and $h(\cdot)$ satisfying Assumption 2 are said to belong to the sector $\left[X_{1}, X_{2}\right]$, $\left[Y_{1}, Y_{2}\right]$ and $\left[H_{1}, H_{2}\right]$, respectively. Note that the sector-bounded nonlinearity of stochastic systems has been studied by $[33,34]$. It should be pointed out that such a nonlinear condition is more general than the usual Lipschitz conditions that have been widely used in $[16,38]$. 
Assumption 3: [20] The distributed time-delay kernel $\kappa(\cdot):[0,+\infty) \rightarrow[0,+\infty)$ is continuous, integrable and also satisfies

$$
\tilde{\kappa}=\int_{0}^{+\infty} \kappa(s) d s<+\infty, \quad \breve{\kappa}=\int_{0}^{+\infty} s \kappa(s) d s<+\infty
$$

Assumption 4: The noise intensity function vector $\sigma_{i}: \mathbb{R} \times \mathbb{R}^{n} \times \mathbb{R}^{n} \rightarrow \mathbb{R}^{n}$ satisfies the Lipschitz condition, i.e., there exist constant matrices $\Sigma_{1}$ and $\Sigma_{2}$ of appropriate dimensions such that the following inequality

$$
\left(\sigma_{i}\left(t, u_{1}, v_{1}\right)-\sigma_{j}\left(t, u_{2}, v_{2}\right)\right)^{T}\left(\sigma_{i}\left(t, u_{1}, v_{1}\right)-\sigma_{j}\left(t, u_{2}, v_{2}\right)\right) \leq\left\|\Sigma_{1}\left(u_{1}-u_{2}\right)\right\|^{2}+\left\|\Sigma_{2}\left(v_{1}-v_{2}\right)\right\|^{2}
$$

holds for all $i, j=1,2, \ldots, N$ and $u_{1}, v_{1}, u_{2}, v_{2} \in \mathbb{R}^{n}$.

By utilizing the Kronecker product ' $\otimes$ ' of matrices, the network system (1) can be written in a compact form as

$$
\begin{aligned}
d x(t)= & {\left[\left(I_{N} \otimes A+W^{(1)} \otimes \Gamma_{1}\right) x(t)+\left(I_{N} \otimes D+W^{(2)} \otimes \Gamma_{2}\right) x(t-\tau(t))+\delta(t)\left(I_{N} \otimes B_{1}\right) F(x(t))\right.} \\
& \left.+(1-\delta(t))\left(I_{N} \otimes B_{2}\right) G(x(t))+\left(I_{N} \otimes C\right) \int_{-\infty}^{t} \kappa(t-s) H(x(s)) d s\right] d t \\
& +\left(W^{(1)} \otimes \Gamma_{1}\right) x(t) d \omega_{1}(t)+\left(W^{(2)} \otimes \Gamma_{2}\right) x(t-\tau(t)) d \omega_{2}(t)+\sigma(t, x(t), x(t-\tau(t))) d \omega_{3}(t),
\end{aligned}
$$

where

$$
\begin{aligned}
x(t) & =\operatorname{col}\left\{x_{1}(t), x_{2}(t), \ldots, x_{N}(t)\right\} \\
F(x(t)) & =\operatorname{col}\left\{f\left(x_{1}(t)\right), f\left(x_{2}(t)\right), \ldots, f\left(x_{N}(t)\right)\right\} \\
G(x(t)) & =\operatorname{col}\left\{g\left(x_{1}(t)\right), g\left(x_{2}(t)\right), \ldots, g\left(x_{N}(t)\right\}\right. \\
H(x(t)) & =\operatorname{col}\left\{h\left(x_{1}(t)\right), h\left(x_{2}(t)\right), \ldots, h\left(x_{N}(t)\right)\right\} \\
\sigma(t, x(t), x(t-\tau(t))) & =\operatorname{col}\left\{\sigma_{1}\left(t, x_{1}(t), x_{1}(t-\tau(t))\right), \cdots, \sigma_{N}\left(t, x_{N}(t), x_{N}(t-\tau(t))\right)\right\} .
\end{aligned}
$$

Let the network (1) (or (12)) be supplemented with initial conditions of the form

$$
x_{i}(s)=\varphi_{i}(s) \in L_{\mathcal{F}_{0}}^{2}\left((-\infty, 0], \mathbb{R}^{n}\right), i=1,2, \cdots, N,
$$

in which $L_{\mathcal{F}_{0}}^{2}\left((-\infty, 0], \mathbb{R}^{n}\right)$ is the family of all $\mathcal{F}_{0}$-measurable $\mathcal{C}\left((-\infty, 0], \mathbb{R}^{n}\right)$-valued random variables which satisfying $\sup _{-\infty \leq s \leq 0} \mathbb{E}\left\{\left\|\varphi_{i}(s)\right\|^{2}\right\}<\infty[23,36]$.

Before stating the main results, a definition and some lemmas are introduced here.

Definition 1: The complex networks (1) (or (12)) is said to be globally asymptotically synchronized in the mean square if

$$
\lim _{t \rightarrow \infty} \mathbb{E}\left\{\left\|x_{i}\left(t, \varphi_{i}(s)\right)-x_{j}\left(t, \varphi_{j}(s)\right)\right\|^{2}\right\}=0
$$

holds for any $i, j \in\{1,2, \ldots, N\}$.

Lemma 1: [13] The Kronecker product has the following properties:

(1) $(\alpha A) \otimes B=A \otimes(\alpha B)$

(2) $(A+B) \otimes C=A \otimes C+B \otimes C$

(3) $(A \otimes B)(C \otimes D)=(A C) \otimes(B D)$;

(4) $(A \otimes B)^{T}=A^{T} \otimes B^{T}$.

Lemma 2: [3] Given any real matrices $\Omega_{1}, \Omega_{2}, \Lambda$ of appropriate dimensions and a number $\varepsilon>0$ such that $\Lambda>0$, then the following inequality holds:

$$
\Omega_{1}^{T} \Omega_{2}+\Omega_{2}^{T} \Omega_{1} \leq \varepsilon \Omega_{1}^{T} \Lambda \Omega_{1}+\varepsilon^{-1} \Omega_{2}^{T} \Lambda^{-1} \Omega_{2} .
$$

Lemma 3: Let $\mathcal{U}=\left(\alpha_{i j}\right)_{N \times N}, P \in \mathbb{R}^{n \times n}, x=\operatorname{col}\left\{x_{1}, x_{2}, \ldots, x_{N}\right\}$ where $x_{i}=\operatorname{col}\left\{x_{i 1}, x_{i 2}, \ldots, x_{i n}\right\} \in \mathbb{R}^{n}$ and $y=\operatorname{col}\left\{y_{1}, y_{2}, \ldots, y_{N}\right\}$ where $y_{i}=\operatorname{col}\left\{y_{i 1}, y_{i 2}, \ldots, y_{i n}\right\} \in \mathbb{R}^{n}(k=1,2, \ldots, N)$. If $\mathcal{U}=\mathcal{U}^{T}$ and each row sum of $\mathcal{U}$ is zero, then

$$
x^{T}(\mathcal{U} \otimes P) y=-\sum_{1 \leq i<j \leq N} \alpha_{i j}\left(x_{i}-x_{j}\right)^{T} P\left(y_{i}-y_{j}\right)
$$


Lemma 4: [20] Let $M$ be a positive semi-definite matrix, $\alpha(\cdot):(-\infty, a] \rightarrow[0,+\infty)$ be a scalar function and $\mathcal{F}(\cdot)$ : $(-\infty, a] \rightarrow \mathbb{R}^{n}$ be a vector function. If the integrations concerned are well defined, the following inequality holds:

$$
\left(\int_{-\infty}^{a} \alpha(s) \mathcal{F}(s) d s\right)^{T} M\left(\int_{-\infty}^{a} \alpha(s) \mathcal{F}(s) d s\right) \leq \int_{-\infty}^{a} \alpha(s) d s\left(\int_{-\infty}^{a} \alpha(s) \mathcal{F}^{T}(s) M \mathcal{F}(s) d s\right) .
$$

Remark 4: In the above lemma, if we take

$$
\alpha(t)=\left\{\begin{array}{l}
1: b \leq t \leq a \\
0: \text { otherwise }
\end{array}\right.
$$

we can get the following well-known Jensen inequality [12]:

$$
\left(\int_{b}^{a} \mathcal{F}(s) d s\right)^{T} M\left(\int_{b}^{a} \mathcal{F}(s) d s\right) \leq(a-b)\left(\int_{b}^{a} \mathcal{F}^{T}(s) M \mathcal{F}(s) d s\right) .
$$

Therefore, the Jensen inequality (15) is a special case of the inequality (14). Lemma 4 will be used to deal with the infinite integral such as the unbounded distributed delays considered in this paper.

Lemma 5: [27] Let $f$ be a nonnegative function defined on $[0,+\infty)$. If $f$ is Lebesgue integrable and is uniformly continuous on $[0,+\infty)$, then $\lim _{t \rightarrow+\infty} f(t)=0$.

\section{MAin Results}

In this section, we deal with the globally mean-square synchronization problem of the complex networks (1) (or (12)) with stochastic nonlinearities and multiple stochastic disturbances.

In the following, for simplicity, we denote

$$
\begin{aligned}
& \hat{X}=X_{1}^{T} X_{2}+X_{2}^{T} X_{1}, \check{X}=X_{1}^{T}+X_{2}^{T} ; \quad \hat{Y}=Y_{1}^{T} Y_{2}+Y_{2}^{T} Y_{1}, \\
& \check{Y}=Y_{1}^{T}+Y_{2}^{T}, \quad \hat{H}=H_{1}^{T} H_{2}+H_{2}^{T} H_{1}, \quad \check{H}=H_{1}^{T}+H_{2}^{T} .
\end{aligned}
$$

Rewrite system (12) as

$$
\begin{aligned}
d x(t)=[ & \left(I_{N} \otimes A+W^{(1)} \otimes \Gamma_{1}\right) x(t)+\left(I_{N} \otimes D+W^{(2)} \otimes \Gamma_{2}\right) x(t-\tau(t))+\delta_{0}\left(I_{N} \otimes B_{1}\right) F(x(t)) \\
& +\left(1-\delta_{0}\right)\left(I_{N} \otimes B_{2}\right) G(x(t))+\left(\delta(t)-\delta_{0}\right)\left(\left(I_{N} \otimes B_{1}\right) F(x(t))-\left(I_{N} \otimes B_{2}\right) G(x(t))\right) \\
& \left.+\left(I_{N} \otimes C\right) \int_{-\infty}^{t} \kappa(t-s) H(x(s)) d s\right] d t+\left(W^{(1)} \otimes \Gamma_{1}\right) x(t) d \omega_{1}(t) \\
& +\left(W^{(2)} \otimes \Gamma_{2}\right) x(t-\tau(t)) d \omega_{2}(t)+\sigma(t, x(t), x(t-\tau(t))) d \omega_{3}(t) .
\end{aligned}
$$

In this paper, the discrete time delay we consider exists in an interval $0<\hbar_{1} \leq \tau(t) \leq \hbar_{2}$, that is, the range of the delay varies in an interval for which the lower bound is not restricted to 0 . Let us represent the time delay $\tau(t)$ as two parts: the constant part $\hbar_{1}$ and the time-varying part $d(t)$,

$$
\tau(t)=d(t)+\hbar_{1}, \quad 0 \leq d(t) \leq \hbar_{2}-\hbar_{1}, \quad \dot{d}(t) \leq \mu .
$$

By utilizing the most updated techniques for achieving delay dependence, the idea of 'delay partitioning' $[25,26]$ is now introduced to the constant part $\hbar_{1}$, that is, we divide $\hbar_{1}$ into $r$ equal divisions, where the integer $r \geq 1$ denotes the number of fractions.

Define

$$
y(t)=\mathcal{A} \eta(t),
$$

where

$$
\begin{gathered}
\mathcal{A}=\left[\left(I_{N} \otimes A+W^{(1)} \otimes \Gamma_{1}\right), 0_{N n \times r N n},\left(I_{N} \otimes D+W^{(2)} \otimes \Gamma_{2}\right), 0_{N n \times N n},\right. \\
\left.\delta_{0}\left(I_{N} \otimes B_{1}\right),\left(1-\delta_{0}\right)\left(I_{N} \otimes B_{2}\right), I_{N} \otimes C, 0_{N n \times N n}\right], \\
\eta(t)=\operatorname{col}\left\{x(t), x\left(t-\frac{\hbar_{1}}{r}\right), \cdots, x\left(t-\frac{r-1}{r} \hbar_{1}\right), x\left(t-\hbar_{1}\right), x(t-\tau(t)), x\left(t-\hbar_{2}\right),\right. \\
\left.F(x(t)), G(x(t)), \int_{-\infty}^{t} \kappa(t-s) H(x(s)) d s, y(t)\right\} .
\end{gathered}
$$


Then, the network (12) can be expressed as

$$
d x(t)=\left[y(t)+\left(\delta(t)-\delta_{0}\right) \mathcal{B} \eta(t)\right] d t+\sigma(t) d \omega(t)
$$

where $\mathcal{B}=\left[0_{N n \times(r+3) N n},\left(I_{N} \otimes B_{1}\right),-\left(I_{N} \otimes B_{2}\right), 0_{N n \times 2 N n}\right], d \omega(t)=\operatorname{col}\left\{d \omega_{1}(t), d \omega_{2}(t), d \omega_{3}(t)\right\}, \sigma(t)=\left[\left(W^{(1)} \otimes\right.\right.$ $\left.\left.\Gamma_{1}\right) x(t),\left(W^{(2)} \otimes \Gamma_{2}\right) x(t-\tau(t)), \sigma(t, x(t), x(t-\tau(t)))\right]$.

Theorem 1: Consider the complex network (1) (or (16)) with unbounded distributed delay and a discrete interval time-varying delay $\left(0<\hbar_{1} \leq \tau(t) \leq \hbar_{2}\right)$. For a given integer $r \geq 1$, the asymptotic synchronization in the mean square for (1) (or (16)) can be achieved if there exist matrices $P_{l}>0(l=1,2), Q_{k}>0(k=1,2, \ldots, r+1), R>0, Z>0$, matrices $M_{k}, S$ and positive scalars $\lambda, \alpha, \beta, \gamma$ such that the following LMIs hold for all $1 \leq i<j \leq N$ :

$$
\begin{aligned}
P_{1} & <\lambda I, \\
\Theta_{i j} & =\left[\begin{array}{ccc}
\Xi_{i j} & \tilde{M} & \tilde{M} \\
* & -R & 0 \\
* & * & -Z
\end{array}\right]<0,
\end{aligned}
$$

where

$$
\begin{aligned}
& \Xi_{i j}=W_{Q}^{T} \tilde{Q} W_{Q}+W_{S}^{T} \Pi_{i j} W_{S}, \quad \tilde{M}=\operatorname{diag}\left\{\sqrt{\frac{\hbar_{1}}{r}} M_{1}, \sqrt{\frac{\hbar_{1}}{r}} M_{2}, \ldots, \sqrt{\frac{\hbar_{1}}{r}} M_{r}, 0,0, \sqrt{\hbar_{2}-\hbar_{1}} M_{r+1}, 0,0,0,0,0\right\}, \\
& W_{Q}=\left[\begin{array}{ccc}
I_{r n \times r n} & 0_{r n \times n} & 0_{r n \times 7 n} \\
0_{r n \times n} & I_{r n \times r n} & 0_{r n \times 7 n}
\end{array}\right], \tilde{Q}=\left[\begin{array}{cc}
\mathbf{Q}+\mathbf{M}+\mathbf{M}^{T} & -\mathbf{M} \\
* & -\mathbf{Q}
\end{array}\right], W_{S}=\left[\begin{array}{ccc}
I_{n \times n} & 0_{n \times(r n+7 n)} \\
0_{n \times r n} & I_{n \times n} & 0_{n \times 7 n} \\
0_{n \times(r n+n)} & I_{n \times n} & 0_{n \times 6 n} \\
0_{n \times(r n+2 n)} & I_{n \times n} & 0_{n \times 5 n} \\
0_{n \times(r n+3 n)} & I_{n \times n} & 0_{n \times 4 n} \\
0_{n \times(r n+4 n)} & I_{n \times n} & 0_{n \times 3 n} \\
0_{n \times(r n+5 n)} & I_{n \times n} & 0_{n \times 2 n} \\
0_{n \times(r n+6 n)} & I_{n \times n} & 0_{n \times n} \\
0_{n \times(r n+7 n)} & I_{n \times n}
\end{array}\right], \\
& \Pi_{i j}=\left[\begin{array}{ccccccccc}
\pi_{(1,1)} & 0 & \pi_{(1,3)} & 0 & \alpha \check{X}+\delta_{0} P_{1} B_{1} & \beta \check{Y}+\left(1-\delta_{0}\right) P_{1} B_{2} & \gamma \check{H} & \pi_{(1,8)} & P_{1} C \\
* & 0 & 0 & M_{r+1}^{T} & 0 & 0 & 0 & 0 & 0 \\
* & * & \pi_{(3,3)} & 0 & 0 & 0 & 0 & \pi_{(3,8)} & 0 \\
* & * & * & -2 M_{r+1} & 0 & 0 & 0 & 0 & 0 \\
* & * & * & * & \pi_{(5,5)} & \pi_{(5,6)} & 0 & \delta_{0} B_{1}^{T} S^{T} & 0 \\
* & * & * & * & * & \pi_{(6,6)} & 0 & \left(1-\delta_{0}\right) B_{2}^{T} S^{T} & 0 \\
* & * & * & * & * & * & \pi_{(7,7)} & 0 & 0 \\
* & * & * & * & * & * & * & \hbar_{2} R-S-S^{T} & S C \\
* & * & * & * & * & * & * & * & -\frac{1}{\tilde{\kappa}} P_{2}
\end{array}\right], \\
& \pi_{(1,1)}=\left(A^{T} P_{1}+P_{1} A\right)+\lambda \Sigma_{1}^{T} \Sigma_{1}+Q_{r+1}-N w_{i j}^{(1,1)} \Gamma_{1}^{T} P_{1} \Gamma_{1}-N w_{i j}^{(1)}\left(P_{1} \Gamma_{1}+\Gamma_{1}^{T} P_{1}\right)-\alpha \hat{X}-\beta \hat{Y}-\gamma \hat{H}, \\
& \pi_{(1,3)}=P_{1} D-N w_{i j}^{(2)} P_{1} \Gamma_{2}, \quad \pi_{(1,8)}=A^{T} S^{T}-N w_{i j}^{(1)} \Gamma_{1}^{T} S^{T}, \\
& \pi_{(3,3)}=\lambda \Sigma_{2}^{T} \Sigma_{2}-(1-\mu) Q_{r+1}-N w_{i j}^{(2,2)} \Gamma_{2}^{T} P_{1} \Gamma_{2}, \quad \pi_{(3,8)}=D^{T} S^{T}-N w_{i j}^{(2)} \Gamma_{2}^{T} S^{T}, \\
& \pi_{(5,5)}=-2 \alpha I+\delta_{0}\left(1-\delta_{0}\right) \hbar_{2} B_{1}^{T} Z B_{1}, \quad \pi_{(5,6)}=-\delta_{0}\left(1-\delta_{0}\right) \hbar_{2} B_{1}^{T} Z B_{2}, \\
& \pi_{(6,6)}=-2 \beta I+\delta_{0}\left(1-\delta_{0}\right) \hbar_{2} B_{2}^{T} Z B_{2}, \quad \pi_{(7,7)}=-2 \gamma I+\tilde{\kappa} P_{2}, \\
& \mathbf{Q}=\operatorname{diag}\left\{Q_{1}, Q_{2}, \ldots, Q_{r}\right\}, \quad \mathbf{M}=\operatorname{diag}\left\{M_{1}, M_{2}, \ldots, M_{r}\right\} \text {, } \\
& \text { and } W^{(1,1)}=W^{(1)} W^{(1)}=\left(w_{i j}^{(1,1)}\right)_{N \times N}, W^{(2,2)}=W^{(2)} W^{(2)}=\left(w_{i j}^{(2,2)}\right)_{N \times N} \text {. }
\end{aligned}
$$

Proof: Construct a matrix functional $V\left(t, x_{t}\right) \in \mathcal{C}^{1,2}\left(\mathbb{R} \times \mathbb{R}^{n N}, \mathbb{R}^{+}\right)$as follows

$$
V\left(t, x_{t}\right)=V_{1}\left(t, x_{t}\right)+V_{2}\left(t, x_{t}\right)+V_{3}\left(t, x_{t}\right)+V_{4}\left(t, x_{t}\right)+V_{5}\left(t, x_{t}\right)+V_{6}\left(t, x_{t}\right),
$$


where

$$
\begin{aligned}
& V_{1}\left(t, x_{t}\right)=x^{T}(t)\left(\mathcal{U} \otimes P_{1}\right) x(t), \\
& V_{2}\left(t, x_{t}\right)=\sum_{k=1}^{r} \int_{t-\frac{k}{r} \hbar_{1}}^{t-\frac{k-1}{r} \hbar_{1}} x^{T}(s)\left(\mathcal{U} \otimes Q_{k}\right) x(s) d s \\
& V_{3}\left(t, x_{t}\right)=\int_{t-\tau(t)}^{t} x^{T}(s)\left(\mathcal{U} \otimes Q_{r+1}\right) x(s) d s \\
& V_{4}\left(t, x_{t}\right)=\int_{0}^{+\infty} \kappa(s) d s \int_{t-s}^{t} H^{T}(x(\theta))\left(\mathcal{U} \otimes P_{2}\right) H(x(\theta)) d \theta, \\
& V_{5}\left(t, x_{t}\right)=\int_{t-\hbar_{2}}^{t} \int_{s}^{t} y^{T}(\theta)(\mathcal{U} \otimes R) y(\theta) d \theta d s, \\
& V_{6}\left(t, x_{t}\right)=\delta_{0}\left(1-\delta_{0}\right) \int_{t-\hbar_{2}}^{t} \int_{s}^{t} \eta^{T}(\theta) \mathcal{B}^{T}(\mathcal{U} \otimes Z) \mathcal{B} \eta(\theta) d \theta d s
\end{aligned}
$$

with $r \geq 1$ (number of fractions) being an integer and $\mathcal{U}=\left[u_{m \alpha}\right]_{N \times N}$ with $u_{m \alpha}= \begin{cases}-1, & \alpha \neq m ; \\ N-1, & \alpha=m .\end{cases}$

The infinitesimal operator $\mathscr{L}$ of $V\left(t, x_{t}\right)$ is defined as follows [24]:

$$
\mathscr{L} V\left(t, x_{t}\right)=\lim _{\Delta \rightarrow 0^{+}} \frac{1}{\Delta}\left[\mathbb{E}\left\{V\left(t+\Delta, x_{t+\Delta}\right) \mid x_{t}\right\}-V\left(t, x_{t}\right)\right] .
$$

With the detailed mathematical derivations in Appendix, we obtain

$$
\mathbb{E}\left\{\mathscr{L} V\left(t, x_{t}\right)\right\}=\sum_{1 \leq i<j \leq N} \xi_{i j}^{T}(t)\left(\Xi_{i j}+\tilde{M}\left(I_{8+r} \otimes\left(R^{-1}+Z^{-1}\right)\right) \tilde{M}^{T}\right) \xi_{i j}(t),
$$

where

$$
\begin{aligned}
\xi_{i j}(t) & =\operatorname{col}\left\{\left(\Upsilon_{i}(t)-\Upsilon_{j}(t)\right),\left(\zeta_{i}(t)-\zeta_{j}(t)\right),\left(\varsigma_{i}(t)-\varsigma_{j}(t)\right)\right\}, \\
\Upsilon_{i}(t) & =\operatorname{col}\left\{x_{i}(t), x_{i}\left(t-\frac{1}{r} \hbar_{1}\right), \ldots, x_{i}\left(t-\frac{r-1}{r} \hbar_{1}\right)\right\}, \\
\zeta_{i}(t) & =\operatorname{col}\left\{x_{i}\left(t-\hbar_{1}\right), x_{i}(t-\tau(t)), x_{i}\left(t-\hbar_{2}\right)\right\}, \\
\varsigma_{i}(t) & =\operatorname{col}\left\{f\left(x_{i}(t)\right), g\left(x_{i}(t)\right), h\left(x_{i}(t)\right), y_{i}(t), \int_{-\infty}^{t} \kappa(t-s) h\left(x_{i}(s)\right) d s\right\} .
\end{aligned}
$$

By the Schur complement, we can see that condition (21) ensures $\hat{\Theta}_{i j}=\Xi_{i j}+\tilde{M}\left(I_{8+r} \otimes\left(R^{-1}+Z^{-1}\right)\right) \tilde{M}^{T}<0$, and then it follows that

$$
\begin{aligned}
\mathbb{E}\left\{\mathscr{L} V\left(t, x_{t}\right)\right\} & \leq \lambda_{\max }\left(\hat{\Theta}_{i j}\right) \sum_{1 \leq i<j \leq N} \mathbb{E}\left\{\left\|\xi_{i j}(t)\right\|^{2}\right\} \\
& \leq \vartheta \sum_{1 \leq i<j \leq N} \mathbb{E}\left\{\left\|x_{i}(t)-x_{j}(t)\right\|^{2}\right\},
\end{aligned}
$$

where $\vartheta=\max _{1 \leq i<j \leq N}\left\{\lambda_{\max }\left(\hat{\Theta}_{i j}\right)\right\}<0$. Therefore we have

$$
\begin{aligned}
\mathbb{E}\{V(t)\}-\mathbb{E}\{V(0)\} & =\int_{0}^{t} \mathbb{E}\{\mathscr{L} V(s)\} d s \\
& \leq \vartheta \int_{0}^{t} \sum_{1 \leq i<j \leq N} \mathbb{E}\left\{\left\|x_{i}(s)-x_{j}(s)\right\|^{2}\right\} d s,
\end{aligned}
$$

which implies that

$$
\int_{0}^{t} \sum_{1 \leq i<j \leq N} \mathbb{E}\left\{\left\|x_{i}(s)-x_{j}(s)\right\|^{2}\right\} d s \leq-\frac{1}{\vartheta} \mathbb{E}\{V(0)\}+\frac{1}{\vartheta} \mathbb{E}\{V(t)\} \leq-\frac{1}{\vartheta} \mathbb{E}\{V(0)\} .
$$


From (8)-(10), it can be inferred that there exists a positive constant $\varepsilon$ such that

$$
\|f(u)-f(v)\| \leq \varepsilon\|u-v\|,\|g(u)-g(v)\| \leq \varepsilon\|u-v\| \text { and }\|h(u)-h(v)\| \leq \varepsilon\|u-v\|, \quad \forall u, v \in \mathbb{R}^{n} .
$$

Accordingly, by (28) and Assumption 3, we can obtain $V(0)<+\infty$, then we have

$$
\int_{0}^{t} \sum_{1 \leq i<j \leq N} \mathbb{E}\left\{\left\|x_{i}(s)-x_{j}(s)\right\|^{2}\right\} d s<+\infty .
$$

Moreover, it is not difficult to see that $\mathbb{E}\left\{\left\|x_{i}(s)-x_{j}(s)\right\|^{2}\right\}$ is uniformly continuous on $[0,+\infty)$. Therefore, by Lemma 5 , we obtain

$$
\sum_{1 \leq i<j \leq N} \mathbb{E}\left\{\left\|x_{i}(s)-x_{j}(s)\right\|^{2}\right\} \rightarrow 0, \text { as } t \rightarrow+\infty
$$

In other words, all the subsystems in (1) are asymptotically synchronized in the mean square. The proof is complete.

Remark 5: The complex dynamical network (1) is a rather general system, since the mixed time delays, the stochastic nonlinearities as well as the stochastic disturbances have been all taken into account in the proposed network framework. It should also be pointed out that the distributed delays studied here comprise two types, one is the unbounded distributed time delay that we have already considered, and the other is the bounded delay that can be demonstrated as follows. If we take

$$
\kappa(s)= \begin{cases}1, & 0 \leq s \leq \rho<+\infty \\ 0, & \text { otherwise }\end{cases}
$$

then the distributed time delay becomes finite in this case. Similar result can be derived easily for the bounded delay case, which are therefore omitted here. Note that, in $[19,28]$, the bounded distributed time delays in neural networks were studied. However, in almost all literature concerning complex (neural) networks with mixed time delays, the stochastic nonlinearities have not been investigated yet. Therefore, in this paper, the model we put forward can reflect more intrinsic characteristics of the real-time systems, and the main results obtained are more general than the existing ones.

Remark 6: By taking advantage of a novel matrix functional and linear matrix inequalities (LMI) techniques, the synchronization criteria have been derived in the form of LMIs for the stochastic complex networks with mixed time delays, stochastic nonlinearities as well as multiple stochastic disturbances. The LMI-based conditions can be readily checked by using the LMI toolbox in Matlab or other standard numerical software. An important feature of the reported results lies in that all the conditions are dependent on both the lower and upper bounds of the time-varying delays, which is made possible by utilizing the most updated techniques for achieving delay dependence.

\section{Numerical Example}

In this section, we present a numerical example so as to illustrate the advantage and usefulness of our main results.

Consider a system coupled by three identical second-order complex networks with the network parameters given as follows:

$$
\begin{gathered}
A=\left[\begin{array}{cc}
-2.5 & 0.3 \\
0.9 & -1
\end{array}\right], \quad B_{1}=\left[\begin{array}{cc}
-1.6 & 0.4 \\
0.3 & -0.5
\end{array}\right], \quad B_{2}=\left[\begin{array}{cc}
-1.5 & 0.5 \\
0.8 & -1.5
\end{array}\right], \quad D=\left[\begin{array}{cc}
-2.4 & 0.8 \\
0.4 & -1.6
\end{array}\right], \\
C=\left[\begin{array}{cc}
-2 & 0.6 \\
0.8 & -1.2
\end{array}\right], \quad \delta_{0}=0.56, \kappa(s)=e^{-4 s}, \tau(t)=1+0.5 \sin (2 t) .
\end{gathered}
$$

Choose the coupling matrices $W^{(1)}, W^{(2)}$ and the linking matrices $\Gamma_{1}, \Gamma_{2}$ as

$$
W^{(1)}=\left[\begin{array}{ccc}
-2 & 1 & 1 \\
1 & -2 & 1 \\
1 & 1 & -2
\end{array}\right], W^{(2)}=\left[\begin{array}{ccc}
-3 & 1 & 2 \\
2 & -3 & 1 \\
1 & 2 & -3
\end{array}\right] ; \Gamma_{1}=\left[\begin{array}{cc}
0.7 & -0.6 \\
-0.4 & 0.7
\end{array}\right] ; \Gamma_{2}=\left[\begin{array}{cc}
-0.3 & 0.25 \\
-0.35 & -0.4
\end{array}\right] .
$$

Let the nonlinear function and the noise intensity function vector be given by

$$
\begin{aligned}
& f\left(x_{i}(t)\right)=\left(0.5 x_{i 1}(t)-\tanh \left(0.2 x_{i 1}(t)\right)+0.2 x_{i 2}(t), 0.95 x_{i 2}(t)-\tanh \left(0.75 x_{i 2}(t)\right)\right)^{T}, \\
& g\left(x_{i}(t)\right)=h\left(x_{i}(t)\right)=\left(0.2 x_{i 1}(t)-\tanh \left(0.1 x_{i 1}(t)\right), 0.1 x_{i 2}(t)\right)^{T}, i=1,2,3 \\
& \sigma(t, u, v)=\left[\begin{array}{cccc}
-0.1 & 0.1 & 0.2 & -0.2 \\
0.1 & -0.1 & 0.2 & -0.2
\end{array}\right]\left[\begin{array}{l}
u \\
v
\end{array}\right] .
\end{aligned}
$$


Then, it is easy to verify that $\tilde{\kappa}=0.25, \mu=1, \hbar_{1}=0.5, \hbar_{2}=1.5$ and

$$
\begin{aligned}
& X_{1}=\left(\begin{array}{cc}
0.3 & 0.2 \\
0 & 0.2
\end{array}\right), X_{2}=\left(\begin{array}{cc}
0.5 & 0.2 \\
0 & 0.95
\end{array}\right), Y_{1}=H_{1}=\left(\begin{array}{cc}
0.1 & 0 \\
0 & 0.1
\end{array}\right), \\
& Y_{2}=H_{2}=\left(\begin{array}{cc}
0.2 & 0 \\
0 & 0.1
\end{array}\right), \Sigma_{1}=\left[\begin{array}{cc}
-0.1 & 0.1 \\
0.1 & -0.1
\end{array}\right], \Sigma_{2}=\left[\begin{array}{cc}
0.2 & -0.2 \\
0.2 & -0.2
\end{array}\right] .
\end{aligned}
$$

By using the Matlab LMI Toolbox, if we take $r=1$, LMIs (20)-(21) are feasible and the solutions are given as:

$$
\begin{aligned}
& P_{1}=\left[\begin{array}{cc}
26.0331 & 7.8656 \\
7.8656 & 15.4338
\end{array}\right], P_{2}=\left[\begin{array}{cc}
17.9620 & -2.9178 \\
-2.9178 & 10.8588
\end{array}\right], Q_{1}=\left[\begin{array}{cc}
24.3735 & -2.2467 \\
-2.2467 & 19.2441
\end{array}\right], \\
& Q_{2}=\left[\begin{array}{cc}
51.0797 & -16.0797 \\
-16.0797 & 21.4102
\end{array}\right], R=\left[\begin{array}{cc}
5.6141 & 3.7871 \\
3.7871 & 4.0428
\end{array}\right], Z=\left[\begin{array}{cc}
14.6002 & 2.9157 \\
2.9157 & 17.2751
\end{array}\right], \\
& \lambda=31.4146, \alpha=19.8248, \beta=24.6340, \gamma=12.2727 .
\end{aligned}
$$

According to Theorem 1, the array of coupled delayed complex networks (1) with multiple stochastic disturbances and stochastic nonlinearities can achieve globally asymptotic synchronization in the mean square under the allowable interval delay. Such a conclusion is further supported by the simulation results given in Fig. 1-Fig. 2, where the initial states for system $(1)$ are taken randomly constants in $[-1,1] \times[-1,1]$.

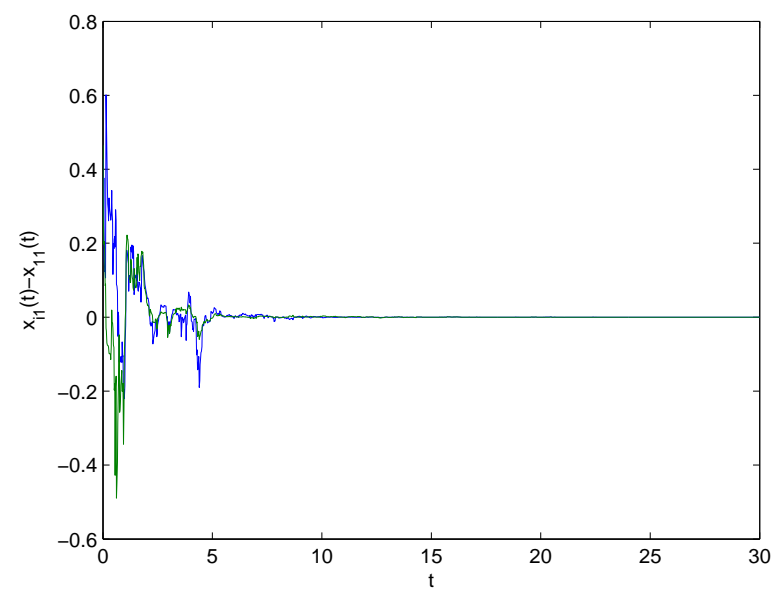

Fig. 1. Synchronization error of $x_{i 1}(t)-x_{11}(t)(i=2,3)$

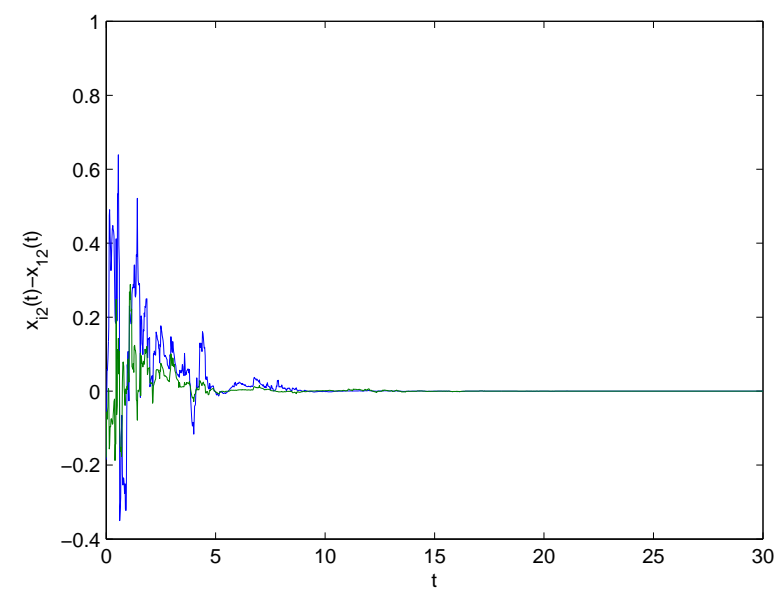

Fig. 2. Synchronization error of $x_{i 2}(t)-x_{12}(t)(i=2,3)$

\section{Conclusions}

In this paper, we have investigated the networks synchronization problem of a new class of stochastic delayed complex networks with $N$ identical subsystems. By employing a novel matrix functional, the properties of Kronecker product, linear matrix inequalities (LMI) techniques and stochastic analysis theory, the synchronization criteria have been established for a stochastic complex networks with mixed time delays, stochastic nonlinearities as well as multiple stochastic disturbances. Note that the LMI-based criteria reported in the present paper are dependent on the allowable lower and upper bound of the discrete time-varying delays, which is made possible by utilizing the most updated techniques for achieving delay dependence. Moreover, the LMI-based criteria can be readily verified by the standard numerical software. In the end of the paper, we have exploited a numerical example to show the usefulness of our results.

Appendix. The detailed derivations of $\mathbb{E}\left\{\mathscr{L} V\left(t, x_{t}\right)\right\}$ in the proof of Theorem 1 .

Based on the properties of Kronecker product and Assumption 1, it follows readily that

$$
\mathcal{U} \otimes W^{(i)}=N W^{(i)},\left(W^{(i)} \otimes \Gamma_{i}\right)(\mathcal{U} \otimes P)\left(W^{(j)} \otimes \Gamma_{j}\right)=N\left(W^{(i)} W^{(j)}\right) \otimes\left(\Gamma_{i} P \Gamma_{j}\right) \quad i, j=1,2 .
$$


Then, with Lemmas 1, 3 and 4, we have

$$
\begin{aligned}
& \mathscr{L} V_{1}\left(t, x_{t}\right)=2 x^{T}(t)\left(\mathcal{U} \otimes P_{1}\right) y(t)+x^{T}(t)\left(W^{(1)} \otimes \Gamma_{1}^{T}\right)\left(\mathcal{U} \otimes P_{1}\right)\left(W^{(1)} \otimes \Gamma_{1}\right) x(t) \\
& +x^{T}(t-\tau(t))\left(W^{(2)} \otimes \Gamma_{2}^{T}\right)\left(\mathcal{U} \otimes P_{1}\right)\left(W^{(2)} \otimes \Gamma_{2}\right) x(t-\tau(t)) \\
& +\sigma^{T}(t, x(t), x(t-\tau(t)))\left(\mathcal{U} \otimes P_{1}\right) \sigma(t, x(t), x(t-\tau(t)))+2\left(\delta(t)-\delta_{0}\right) x^{T}(t)\left(\mathcal{U} \otimes P_{1}\right) \mathcal{B} \eta(t) \\
& =\sum_{1 \leq i<j \leq N}\left[\left(x_{i}(t)-x_{j}(t)\right)^{T}\left(2 P_{1}\left(y_{i}(t)-y_{j}(t)\right)-N w_{i j}^{(1,1)} \Gamma_{1}^{T} P_{1} \Gamma_{1}\left(x_{i}(t)-x_{j}(t)\right)\right)\right. \\
& -\left(x_{i}(t-\tau(t))-x_{j}(t-\tau(t))\right)^{T} N w_{i j}^{(2,2)} \Gamma_{2}^{T} P_{1} \Gamma_{2}\left(x_{i}(t-\tau(t))-x_{j}(t-\tau(t))\right) \\
& +\left(\sigma_{i}\left(t, x_{i}(t), x_{i}(t-\tau(t))\right)-\sigma_{j}\left(t, x_{j}(t), x_{j}(t-\tau(t))\right)\right)^{T} P_{1} \\
& \left.\times\left(\sigma_{i}\left(t, x_{i}(t), x_{i}(t-\tau(t))\right)-\sigma_{j}\left(t, x_{j}(t), x_{j}(t-\tau(t))\right)\right)\right] \\
& =\sum_{1 \leq i<j \leq N}\left\{( x _ { i } ( t ) - x _ { j } ( t ) ) ^ { T } \left[\left(P_{1} A+A^{T} P_{1}-N w_{i j}^{(1)}\left(P_{1} \Gamma_{1}+\Gamma_{1}^{T} P_{1}\right)\right.\right.\right. \\
& \left.-N w_{i j}^{(1,1)} \Gamma_{1}^{T} P_{1} \Gamma_{1}\right)\left(x_{i}(t)-x_{j}(t)\right)+2\left(P_{1} D-N w_{i j}^{(2)} P_{1} \Gamma_{2}\right)\left(x_{i}(t-\tau(t))-x_{j}(t-\tau(t))\right) \\
& +2 \delta_{0} P_{1} B_{1}\left(f\left(x_{i}(t)\right)-f\left(x_{j}(t)\right)\right)+2\left(1-\delta_{0}\right) P_{1} B_{2}\left(g\left(x_{i}(t)\right)-g\left(x_{j}(t)\right)\right) \\
& \left.+2 P_{1} C \int_{-\infty}^{t} \kappa(t-s)\left(h\left(x_{i}(s)\right)-h\left(x_{j}(s)\right)\right) d s\right] \\
& -\left(x_{i}(t-\tau(t))-x_{j}(t-\tau(t))\right)^{T} N w_{i j}^{(2,2)} \Gamma_{2}^{T} P_{1} \Gamma_{2}\left(x_{i}(t-\tau(t))-x_{j}(t-\tau(t))\right) \\
& +\left(\sigma_{i}\left(t, x_{i}(t), x_{i}(t-\tau(t))\right)-\sigma_{j}\left(t, x_{j}(t), x_{j}(t-\tau(t))\right)\right)^{T} P_{1} \\
& \left.\times\left(\sigma_{i}\left(t, x_{i}(t), x_{i}(t-\tau(t))\right)-\sigma_{j}\left(t, x_{j}(t), x_{j}(t-\tau(t))\right)\right)\right\}, \\
& \mathscr{L} V_{2}\left(t, x_{t}\right)=x^{T}(t)\left(\mathcal{U} \otimes Q_{1}\right) x(t)-x^{T}\left(t-\hbar_{1}\right)\left(\mathcal{U} \otimes Q_{r}\right) x\left(t-\hbar_{1}\right) \\
& -\sum_{l=1}^{r-1}\left(x^{T}\left(t-\frac{l}{r} \hbar_{1}\right)\left(\mathcal{U} \otimes\left(Q_{l}-Q_{l+1}\right)\right) x\left(t-\frac{l}{r} \hbar_{1}\right)\right) \\
& =\sum_{1 \leq i<j \leq N}\left[\left(x_{i}(t)-x_{j}(t)\right)^{T} Q_{1}\left(x_{i}(t)-x_{j}(t)\right)-\left(x_{i}\left(t-\hbar_{1}\right)-x_{j}\left(t-\hbar_{1}\right)\right)^{T}\right. \\
& \times Q_{r}\left(x_{i}\left(t-\hbar_{1}\right)-x_{j}\left(t-\hbar_{1}\right)\right)-\sum_{l=1}^{r-1}\left(x_{i}\left(t-\frac{l}{r} \hbar_{1}\right)-x_{j}\left(t-\frac{l}{r} \hbar_{1}\right)\right)^{T} \\
& \times\left(Q_{l}-Q_{l+1}\right)\left(x_{i}\left(t-\frac{l}{r} \hbar_{1}\right)-x_{j}\left(t-\frac{l}{r} \hbar_{1}\right)\right), \\
& \mathscr{L} V_{3}\left(t, x_{t}\right)=x^{T}(t)\left(\mathcal{U} \otimes Q_{r+1}\right) x(t)-(1-\dot{\tau}(t)) x^{T}(t-\tau(t))\left(\mathcal{U} \otimes Q_{r+1}\right) x(t-\tau(t)) \\
& \leq \sum_{1 \leq i<j \leq N}\left[\left(x_{i}(t)-x_{j}(t)\right)^{T} Q_{r+1}\left(x_{i}(t)-x_{j}(t)\right)\right. \\
& \left.-(1-\mu)\left(x_{i}(t-\tau(t))-x_{j}(t-\tau(t))\right)^{T} Q_{r+1}\left(x_{i}(t-\tau(t))-x_{j}(t-\tau(t))\right)\right], \\
& \mathscr{L} V_{4}\left(t, x_{t}\right)=\int_{0}^{+\infty} \kappa(s) H^{T}(x(t))\left(\mathcal{U} \otimes P_{2}\right) H(x(t)) d s-\int_{0}^{+\infty} \kappa(s) H^{T}(x(t-s))\left(\mathcal{U} \otimes P_{2}\right) H(x(t-s)) d s \\
& =\tilde{\kappa} H^{T}(x(t))\left(\mathcal{U} \otimes P_{2}\right) H(x(t))-\int_{0}^{+\infty} \kappa(s) H^{T}(x(t-s))\left(\mathcal{U} \otimes P_{2}\right) H(x(t-s)) d s \\
& =\sum_{1 \leq i<j \leq N}\left[\tilde{\kappa}\left(h\left(x_{i}(t)\right)-h\left(x_{j}(t)\right)\right)^{T} P_{2}\left(h\left(x_{i}(t)\right)-h\left(x_{j}(t)\right)\right)\right. \\
& \left.-\int_{-\infty}^{t} \kappa(t-s)\left(h\left(x_{i}(s)\right)-h\left(x_{j}(s)\right)\right)^{T} P_{2}\left(h\left(x_{i}(s)\right)-h\left(x_{j}(s)\right)\right) d s\right] \\
& \leq \sum_{1 \leq i<j \leq N}\left[\tilde{\kappa}\left(h\left(x_{i}(t)\right)-h\left(x_{j}(t)\right)\right)^{T} P_{2}\left(h\left(x_{i}(t)\right)-h\left(x_{j}(t)\right)\right)-\frac{1}{\tilde{\kappa}}\left(\int _ { - \infty } ^ { t } \kappa ( t - s ) \left(h\left(x_{i}(s)\right)\right.\right.\right. \\
& \left.\left.\left.-h\left(x_{j}(s)\right)\right) d s\right)^{T} P_{2}\left(\int_{-\infty}^{t} \kappa(t-s)\left(h\left(x_{i}(s)\right)-h\left(x_{j}(s)\right)\right) d s\right)\right] \\
& \mathscr{L} V_{5}\left(t, x_{t}\right)=\hbar_{2} y^{T}(t)(\mathcal{U} \otimes R) y(t)-\int_{t-\hbar_{1}}^{t} y^{T}(s)(\mathcal{U} \otimes R) y(s) d s-\int_{t-\hbar_{2}}^{t-\hbar_{1}} y^{T}(s)(\mathcal{U} \otimes R) y(s) d s \\
& =\sum_{1 \leq i<j \leq N}\left[\hbar_{2}\left(y_{i}(t)-y_{j}(t)\right)^{T} R\left(y_{i}(t)-y_{j}(t)\right)-\sum_{k=1}^{r}\left(\int_{t-\frac{k}{r} \hbar_{1}}^{t-\frac{k-1}{r} \hbar_{1}}\left(y_{i}(s)-y_{j}(s)\right)^{T}\right.\right. \\
& \left.\left.\times R\left(y_{i}(s)-y_{j}(s)\right) d s\right)-\int_{t-\hbar_{2}}^{t-\hbar_{1}}\left(y_{i}(s)-y_{j}(s)\right)^{T} R\left(y_{i}(s)-y_{j}(s)\right) d s\right]
\end{aligned}
$$




$$
\begin{aligned}
\mathscr{L} V_{6}\left(t, x_{t}\right)= & \hbar_{2} \delta_{0}\left(1-\delta_{0}\right) \eta^{T}(t) \mathcal{B}^{T}(\mathcal{U} \otimes Z) \mathcal{B} \eta(t) \\
& -\delta_{0}\left(1-\delta_{0}\right)\left[\int_{t-\hbar_{1}}^{t} \eta^{T}(s) \mathcal{B}^{T}(\mathcal{U} \otimes Z) \mathcal{B} \eta(s) d s+\int_{t-\hbar_{2}}^{t-\hbar_{1}} \eta^{T}(s) \mathcal{B}^{T}(\mathcal{U} \otimes Z) \mathcal{B} \eta(s) d s\right] \\
= & \delta_{0}\left(1-\delta_{0}\right) \sum_{1 \leq i<j \leq N}\left[\hbar _ { 2 } ( \mathcal { B } _ { i } \eta _ { i } ( t ) - \mathcal { B } _ { j } \eta _ { j } ( t ) ) ^ { T } Z \left(\left(\mathcal{B}_{i} \eta_{i}(t)-\mathcal{B}_{j} \eta_{j}(t)\right)\right.\right. \\
& -\sum_{k=1}^{r}\left(\int_{t-\frac{k}{r} \hbar_{1}}^{t-\frac{k-1}{r} \hbar_{1}}\left(\mathcal{B}_{i} \eta_{i}(s)-\mathcal{B}_{j} \eta_{j}(s)\right)^{T} Z\left(\mathcal{B}_{i} \eta_{i}(s)-\mathcal{B}_{j} \eta_{j}(s)\right) d s\right) \\
& \left.-\int_{t-\hbar_{2}}^{t-\hbar_{1}}\left(\mathcal{B}_{i} \eta_{i}(s)-\mathcal{B}_{j} \eta_{j}(s)\right)^{T} Z\left(\mathcal{B}_{i} \eta_{i}(s)-\mathcal{B}_{j} \eta_{j}(s)\right) d s\right] \\
= & \delta_{0}\left(1-\delta_{0}\right) \sum_{1 \leq i<j \leq N}\left[\hbar _ { 2 } \left(\left(f\left(x_{i}(t)\right)-f\left(x_{j}(t)\right)\right)^{T} B_{1}^{T} Z B_{1}\left(f\left(x_{i}(t)\right)-f\left(x_{j}(t)\right)\right)\right.\right. \\
& +\left(g\left(x_{i}(t)\right)-g\left(x_{j}(t)\right)\right)^{T} B_{2}^{T} Z B_{2}\left(g\left(x_{i}(t)\right)-g\left(x_{j}(t)\right)\right) \\
& \left.-2\left(f\left(x_{i}(t)\right)-f\left(x_{j}(t)\right)\right)^{T} B_{1}^{T} Z B_{2}\left(g\left(x_{i}(t)\right)-g\left(x_{j}(t)\right)\right)\right) \\
& -\sum_{k=1}^{r}\left(\int_{t-\frac{k}{r} \hbar_{1}}^{t-\frac{k-1}{r} \hbar_{1}}\left(\mathcal{B}_{i} \eta_{i}(s)-\mathcal{B}_{j} \eta_{j}(s)\right)^{T} Z\left(\mathcal{B}_{i} \eta_{i}(s)-\mathcal{B}_{j} \eta_{j}(s)\right) d s\right) \\
& \left.-\int_{t-\hbar_{2}}^{t-\hbar_{1}}\left(\mathcal{B}_{i} \eta_{i}(s)-\mathcal{B}_{j} \eta_{j}(s)\right)^{T} Z\left(\mathcal{B}_{i} \eta_{i}(s)-\mathcal{B}_{j} \eta_{j}(s)\right) d s\right]
\end{aligned}
$$

where the symbol $\mathcal{B}_{i} \eta_{i}(t)$ means the term $B_{1} f\left(x_{i}(t)\right)-B_{2} g\left(x_{i}(t)\right)$.

From Newton-Leibniz formula, we have that for matrices $M_{k}(k=1,2, \ldots, r)$

$$
\begin{aligned}
& \phi_{k}(t) \triangleq 2 x^{T}\left(t-\frac{k-1}{r} \hbar_{1}\right)\left(\mathcal{U} \otimes M_{k}\right)\left[x\left(t-\frac{k-1}{r} \hbar_{1}\right)-x\left(t-\frac{k}{r} \hbar_{1}\right)-\int_{t-\frac{k}{r} \hbar_{1}}^{t-\frac{k-1}{r} \hbar_{1}} d x(s)\right]=0, \\
& \varphi(t) \triangleq 2 x^{T}\left(t-\hbar_{2}\right)\left(\mathcal{U} \otimes M_{r+1}\right)\left[x\left(t-\hbar_{1}\right)-x\left(t-\hbar_{2}\right)-\int_{t-\hbar_{2}}^{t-\hbar_{1}} d x(s)\right]=0 .
\end{aligned}
$$

Note that, in $\phi_{k}(t)$, it follows from Lemma 2 that for all $k=1,2, \ldots, r$ :

$$
\begin{aligned}
& -2 x^{T}\left(t-\frac{k-1}{r} \hbar_{1}\right)\left(\mathcal{U} \otimes M_{k}\right) \int_{t-\frac{k}{r} \hbar_{1}}^{t-\frac{k-1}{r} \hbar_{1}} d x(s) \\
= & -2 x^{T}\left(t-\frac{k-1}{r} \hbar_{1}\right)\left(\mathcal{U} \otimes M_{k}\right) \int_{t-\frac{k}{r} \hbar_{1}}^{t-\frac{k-1}{r} \hbar_{1}}\left[\left(y(s)+\left(\delta(s)-\delta_{0}\right) \mathcal{B} \eta(s)\right) d s+\sigma(s) d \omega(s)\right] \\
= & \sum_{1 \leq i<j \leq N}\left[-2\left(x_{i}\left(t-\frac{k-1}{r} \hbar_{1}\right)-x_{j}\left(t-\frac{k-1}{r} \hbar_{1}\right)\right)^{T} M_{k}\left(\int_{t-\frac{k}{r} \hbar_{1}}^{t-\frac{k-1}{r} \hbar_{1}}\left(y_{i}(s)-y_{j}(s)\right) d s\right)\right. \\
& \left.-2\left(x_{i}\left(t-\frac{k-1}{r} \hbar_{1}\right)-x_{j}\left(t-\frac{k-1}{r} \hbar_{1}\right)\right)^{T} M_{k}\left(\int_{t-\frac{k}{r} \hbar_{1}}^{t-\frac{k-1}{r} \hbar_{1}}\left(\delta(s)-\delta_{0}\right)\left(\mathcal{B} \eta_{i}(s)-\mathcal{B} \eta_{j}(s)\right) d s\right)\right] \\
& -2 x^{T}\left(t-\frac{k-1}{r} \hbar_{1}\right)\left(\mathcal{U} \otimes M_{k}\right) \int_{t-\frac{k}{r} \hbar_{1}}^{t-\frac{k-1}{r} \hbar_{1}} \sigma(s) d \omega(s) \\
& \sum_{1 \leq i<j \leq N}\left[\frac{1}{r} \hbar_{1}\left(x_{i}\left(t-\frac{k-1}{r} \hbar_{1}\right)-x_{j}\left(t-\frac{k-1}{r} \hbar_{1}\right)\right)^{T} M_{k}\left(R^{-1}+Z^{-1}\right) M_{k}^{T}\right. \\
& \times\left(x_{i}\left(t-\frac{k-1}{r} \hbar_{1}\right)-x_{j}\left(t-\frac{k-1}{r} \hbar_{1}\right)\right)+\left(\int_{t-\frac{k}{r} \hbar_{1}}^{t-\frac{k-1}{r} \hbar_{1}}\left(y_{i}(s)-y_{j}(s)\right)^{T} R\left(y_{i}(s)-y_{j}(s)\right) d s\right) \\
& \left.+\left(\int_{t-\frac{k}{r} \hbar_{1}}^{t-\frac{k-1}{r} \hbar_{1}}\left(\delta(s)-\delta_{0}\right)^{2}\left(\mathcal{B}_{i} \eta_{i}(s)-\mathcal{B}_{j} \eta_{j}(s)\right)^{T} Z\left(\mathcal{B}_{i} \eta_{i}(s)-\mathcal{B}_{j} \eta_{j}(s)\right) d s\right)\right] \\
& -2 x^{T}\left(t-\frac{k-1}{r} \hbar_{1}\right)\left(\mathcal{U} \otimes M_{k}\right) \int_{t-\frac{k}{r} \hbar_{1}}^{t-\frac{k-1}{r} \hbar_{1}} \sigma(s) d \omega(s) .
\end{aligned}
$$


Similarly, in $\varphi(t)$, we can show that

$$
\begin{aligned}
& -2 x^{T}\left(t-\hbar_{2}\right)\left(\mathcal{U} \otimes M_{r+1}\right) \int_{t-\hbar_{2}}^{t-\hbar_{1}} d x(s) \\
= & -2 x^{T}\left(t-\hbar_{2}\right)\left(\mathcal{U} \otimes M_{r+1}\right) \int_{t-\hbar_{2}}^{t-\hbar_{1}}\left[\left(y(s)+\left(\delta(s)-\delta_{0}\right) \mathcal{B} \eta(s)\right) d s+\sigma(s) d \omega(s)\right] \\
\leq & \sum_{1 \leq i<j \leq N}\left[\left(\hbar_{2}-\hbar_{1}\right)\left(x_{i}\left(t-\hbar_{2}\right)-x_{j}\left(t-\hbar_{2}\right)\right)^{T} M_{r+1}\left(R^{-1}+Z^{-1}\right) M_{r+1}^{T}\left(x_{i}\left(t-\hbar_{2}\right)-x_{j}\left(t-\hbar_{2}\right)\right)\right. \\
& +\int_{t-\hbar_{2}}^{t-\hbar_{1}}\left(y_{i}(s)-y_{j}(s)\right)^{T} R\left(y_{i}(s)-y_{j}(s)\right) d s+\int_{t-\hbar_{2}}^{t-\hbar_{1}}\left(\delta(s)-\delta_{0}\right)^{2}\left(\mathcal{B}_{i} \eta_{i}(s)-\mathcal{B}_{j} \eta_{j}(s)\right)^{T} Z \\
& \left.\times\left(\mathcal{B}_{i} \eta_{i}(s)-\mathcal{B}_{j} \eta_{j}(s)\right) d s\right]-2 x^{T}\left(t-\hbar_{2}\right)\left(\mathcal{U} \otimes M_{r+1}\right) \int_{t-\hbar_{2}}^{t-\hbar_{1}} \sigma(s) d \omega(s) .
\end{aligned}
$$

From the definition (18), we have

$$
\begin{aligned}
\psi(t) \triangleq & 2 y^{T}(t)(\mathcal{U} \otimes S)\left[\left(I_{N} \otimes A+W^{(1)} \otimes \Gamma_{1}\right) x(t)+\left(I_{N} \otimes D+W^{(2)} \otimes \Gamma_{2}\right) x(t-\tau(t))+\delta_{0}\left(I_{N} \otimes B_{1}\right)\right. \\
& \left.\times F(x(t))+\left(1-\delta_{0}\right)\left(I_{N} \otimes B_{2}\right) G(x(t))+\left(I_{N} \otimes C\right) \int_{-\infty}^{t} \kappa(t-s) H(x(s)) d s-y(t)\right]=0,
\end{aligned}
$$

which implies that

$$
\begin{aligned}
\psi(t)= & \sum_{1 \leq i<j \leq N}\left[2\left(x_{i}(t)-x_{j}(t)\right)^{T}\left(A^{T} S^{T}-N w_{i j}^{(1)} \Gamma_{1}^{T} S^{T}\right)\left(y_{i}(t)-y_{j}(t)\right)+2\left(x_{i}(t-\tau(t))-x_{j}(t-\tau(t))\right)^{T}\right. \\
& \times\left(D^{T} S^{T}-N w_{i j}^{(2)} \Gamma_{2}^{T} S^{T}\right)\left(y_{i}(t)-y_{j}(t)\right)+2\left(f\left(x_{i}(t)\right)-f\left(x_{j}(t)\right)\right)^{T} \delta_{0} B_{1}^{T} S^{T}\left(y_{i}(t)-y_{j}(t)\right) \\
& +2\left(g\left(x_{i}(t)\right)-g\left(x_{j}(t)\right)\right)^{T}\left(1-\delta_{0}\right) B_{2}^{T} S^{T}\left(y_{i}(t)-y_{j}(t)\right)+2\left(\int_{-\infty}^{t} \kappa(t-s)\left(h\left(x_{i}(s)\right)-h\left(x_{j}(s)\right)\right) d s\right)^{T} \\
& \left.\times C^{T} S^{T}\left(y_{i}(t)-y_{j}(t)\right)-\left(y_{i}(t)-y_{j}(t)\right)^{T}\left(S^{T}+S\right)\left(y_{i}(t)-y_{j}(t)\right)\right]=0 .
\end{aligned}
$$

According to Assumption 4 and condition (20), it is clear that

$$
\begin{aligned}
& {\left[\sigma_{i}\left(t, x_{i}(t), x_{i}(t-\tau(t))\right)-\sigma_{j}\left(t, x_{j}(t), x_{j}(t-\tau(t))\right)\right]^{T} P_{1}\left[\sigma_{i}\left(t, x_{i}(t), x_{i}(t-\tau(t))\right)-\sigma_{j}\left(t, x_{j}(t), x_{j}(t-\tau(t))\right)\right]} \\
& \leq \lambda\left[\left(x_{i}(t)-x_{j}(t)\right)^{T} \Sigma_{1}^{T} \Sigma_{1}\left(x_{i}(t)-x_{j}(t)\right)+\left(x_{i}(t-\tau(t))-x_{j}(t-\tau(t))\right)^{T} \Sigma_{2}^{T} \Sigma_{2}\left(x_{i}(t-\tau(t))-x_{j}(t-\tau(t))\right)\right], \quad 1 \leq i<j \leq N .
\end{aligned}
$$

At the same time, Assumption 2 ensures that the following inequality holds:

$$
\alpha\left[\begin{array}{c}
x_{i}(t)-x_{j}(t) \\
f\left(x_{i}(t)\right)-f\left(x_{j}(t)\right)
\end{array}\right]^{T}\left[\begin{array}{cc}
X_{1}^{T} X_{2}+X_{2}^{T} X_{1} & -\left(X_{1}^{T}+X_{2}^{T}\right) \\
* & 2 I
\end{array}\right]\left[\begin{array}{c}
x_{i}(t)-x_{j}(t) \\
f\left(x_{i}(t)\right)-f\left(x_{j}(t)\right)
\end{array}\right] \leq 0
$$

i.e.,

$$
\alpha\left[\begin{array}{c}
x_{i}(t)-x_{j}(t) \\
f\left(x_{i}(t)\right)-f\left(x_{j}(t)\right)
\end{array}\right]^{T}\left[\begin{array}{cc}
\hat{X} & -\check{X} \\
* & 2 I
\end{array}\right]\left[\begin{array}{c}
x_{i}(t)-x_{j}(t) \\
f\left(x_{i}(t)\right)-f\left(x_{j}(t)\right)
\end{array}\right] \leq 0, \quad 1 \leq i<j \leq N .
$$

Similarly, one has

$$
\begin{gathered}
\beta\left[\begin{array}{c}
x_{i}(t)-x_{j}(t) \\
g\left(x_{i}(t)\right)-g\left(x_{j}(t)\right)
\end{array}\right]^{T}\left[\begin{array}{cc}
\hat{Y} & -\check{Y} \\
* & 2 I
\end{array}\right]\left[\begin{array}{c}
x_{i}(t)-x_{j}(t) \\
g\left(x_{i}(t)\right)-g\left(x_{j}(t)\right)
\end{array}\right] \leq 0, \\
\gamma\left[\begin{array}{c}
x_{i}(t)-x_{j}(t) \\
h\left(x_{i}(t)\right)-h\left(x_{j}(t)\right)
\end{array}\right]^{T}\left[\begin{array}{cc}
\hat{H} & -\check{H} \\
* & 2 I
\end{array}\right]\left[\begin{array}{c}
x_{i}(t)-x_{j}(t) \\
h\left(x_{i}(t)\right)-h\left(x_{j}(t)\right)
\end{array}\right] \leq 0 .
\end{gathered}
$$

Combining (29)-(44), considering condition (6) and noting that

$$
\begin{aligned}
& \mathbb{E}\left\{2 x^{T}\left(t-\hbar_{2}\right)\left(\mathcal{U} \otimes M_{r+1}\right) \int_{t-\hbar_{2}}^{t-\hbar_{1}} \sigma(s) d \omega(s)\right\}=0, \\
& \mathbb{E}\left\{2 x^{T}\left(t-\frac{k-1}{r} \hbar_{1}\right)\left(\mathcal{U} \otimes M_{k}\right) \int_{t-\frac{k}{r} \hbar_{1}}^{t-\frac{k-1}{r} \hbar_{1}} \sigma(s) d \omega(s)\right\}=0 \quad(k=1,2, \ldots, r) ;
\end{aligned}
$$


we get

$$
\begin{aligned}
& \mathbb{E}\left\{\mathscr{L} V\left(t, x_{t}\right)\right\} \\
& \leq \sum_{1 \leq i<j \leq N} \mathbb{E}\left\{( x _ { i } ( t ) - x _ { j } ( t ) ) ^ { T } \left[\left(P_{1} A+A^{T} P_{1}-N w_{i j}^{(1)}\left(\Gamma_{1}^{T} P_{1}+P_{1} \Gamma_{1}\right)+\lambda \Sigma_{1}^{T} \Sigma_{1}+Q_{r+1}-\alpha \hat{X}-\beta \hat{Y}-\gamma \hat{H}\right.\right.\right. \\
& \left.-N w_{i j}^{(1,1)} \Gamma_{1}^{T} P_{1} \Gamma_{1}\right)\left(x_{i}(t)-x_{j}(t)\right)+\left(2\left(P_{1} D-N w_{i j}^{(2)} P_{1} \Gamma_{2}\right)\left(x_{i}(t-\tau(t))-x_{j}(t-\tau(t))\right)+2\left(\alpha \check{X}+\delta_{0} P_{1} B_{1}\right)\right. \\
& \left(f\left(x_{i}(t)\right)-f\left(x_{j}(t)\right)\right)+2\left(\beta \check{Y}+\left(1-\delta_{0} P_{1} B_{2}\right)\right)\left(g\left(x_{i}(t)\right)-g\left(x_{j}(t)\right)\right)+2 \gamma \check{H}\left(h\left(x_{i}(t)\right)-h\left(x_{j}(t)\right)\right)+\left(2 A^{T} S^{T}\right. \\
& \left.\left.\left.-2 N w_{i j}^{(1)} \Gamma_{1}^{T} S^{T}\right)\left(y_{i}(t)-y_{j}(t)\right)+2 P_{1} C \int_{-\infty}^{t} \kappa(t-s)\left(h\left(x_{i}(s)\right)-h\left(x_{j}(s)\right)\right) d s\right)\right]+\left(x_{i}(t-\tau(t))\right. \\
& \left.-x_{j}(t-\tau(t))\right)^{T}\left[\left(\lambda \Sigma_{2}^{T} \Sigma_{2}-(1-\mu) Q_{r+1}-N w_{i j}^{(2,2)} \Gamma_{2}^{T} P_{1} \Gamma_{2}\right)\left(x_{i}(t-\tau(t))-x_{j}(t-\tau(t))\right)+\left(2 D^{T} S^{T}\right.\right. \\
& \left.\left.-2 N w_{i j}^{(2)} \Gamma_{2}^{T} S^{T}\right)\left(y_{i}(t)-y_{j}(t)\right)\right]+\left(f\left(x_{i}(t)\right)-f\left(x_{j}(t)\right)\right)^{T}\left[\left(-2 \alpha I+\delta_{0}\left(1-\delta_{0}\right) \hbar_{2} B_{1}^{T} Z B_{1}\right)\right. \\
& \left.\left(f\left(x_{i}(t)\right)-f\left(x_{j}(t)\right)\right)+2 \delta_{0} B_{1}^{T} S^{T}\left(y_{i}(t)-y_{j}(t)\right)-2 \delta_{0}\left(1-\delta_{0}\right) \hbar_{2} B_{1}^{T} Z B_{2}\left(g\left(x_{i}(t)\right)-g\left(x_{j}(t)\right)\right)\right] \\
& +\left(g\left(x_{i}(t)\right)-g\left(x_{j}(t)\right)\right)^{T}\left[\left(-2 \beta I+\delta_{0}\left(1-\delta_{0}\right) \hbar_{2} B_{2}^{T} Z B_{2}\right)\left(g\left(x_{i}(t)\right)-g\left(x_{j}(t)\right)\right)+2\left(1-\delta_{0}\right) B_{2}^{T} S^{T}\right. \\
& \left.\left(y_{i}(t)-y_{j}(t)\right)\right]+\left(h\left(x_{i}(t)\right)-h\left(x_{j}(t)\right)\right)^{T}\left(-2 \gamma I+\tilde{\kappa} P_{2}\right)\left(h\left(x_{i}(t)\right)-h\left(x_{j}(t)\right)\right)+ \\
& \left(\int_{-\infty}^{t} \kappa(t-s)\left(h\left(x_{i}(s)\right)-h\left(x_{j}(s)\right)\right) d s\right)^{T}\left[2 C^{T} S^{T}\left(y_{i}(t)-y_{j}(t)\right)-\frac{1}{\tilde{\kappa}} P_{2} \int_{-\infty}^{t} \kappa(t-s)\left(h\left(x_{i}(s)\right)\right.\right. \\
& \left.\left.-h\left(x_{j}(s)\right)\right) d s\right]+\left(y_{i}(t)-y_{j}(t)\right)^{T}\left(\hbar_{2} R-S-S^{T}\right)\left(y_{i}(t)-y_{j}(t)\right) \\
& +\left(\Upsilon_{i}(t)-\Upsilon_{j}(t)\right)^{T}\left[\left(\mathbf{Q}+\mathbf{M}+\mathbf{M}^{T}\right)\left(\Upsilon_{i}(t)-\Upsilon_{j}(t)\right)-2 \mathbf{M}\left(\Upsilon_{i}\left(t-\frac{1}{r} \hbar_{1}\right)-\Upsilon_{j}\left(t-\frac{1}{r} \hbar_{1}\right)\right)\right] \\
& -\left(\Upsilon_{i}\left(t-\frac{1}{r} \hbar_{1}\right)-\Upsilon_{j}\left(t-\frac{1}{r} \hbar_{1}\right)\right)^{T} \mathbf{Q}\left(\Upsilon_{i}\left(t-\frac{1}{r} \hbar_{1}\right)-\Upsilon_{j}\left(t-\frac{1}{r} \hbar_{1}\right)\right) \\
& +2\left(x_{i}\left(t-\hbar_{2}\right)-x_{j}\left(t-\hbar_{2}\right)\right)^{T} M_{r+1}\left[\left(x_{i}\left(t-\hbar_{1}\right)-x_{j}\left(t-\hbar_{1}\right)\right)-\left(x_{i}\left(t-\hbar_{2}\right)-x_{j}\left(t-\hbar_{2}\right)\right)\right] \\
& +\frac{1}{r} \hbar_{1}\left(\Upsilon_{i}(t)-\Upsilon_{j}(t)\right)^{T} \mathbf{M}\left(I_{r} \otimes\left(R^{-1}+Z^{-1}\right)\right) \mathbf{M}^{T}\left(\Upsilon_{i}(t)-\Upsilon_{j}(t)\right)+\left(\hbar_{2}-\hbar_{1}\right)\left(x_{i}\left(t-\hbar_{2}\right)-x_{j}\left(t-\hbar_{2}\right)\right)^{T} \\
& \left.M_{r+1}\left(R^{-1}+Z^{-1}\right) M_{r+1}^{T}\left(x_{i}\left(t-\hbar_{2}\right)-x_{j}\left(t-\hbar_{2}\right)\right)\right\} \\
& =\sum_{1 \leq i<j \leq N} \xi_{i j}^{T}(t)\left(\Xi_{i j}+\tilde{M}\left(I_{8+r} \otimes\left(R^{-1}+Z^{-1}\right)\right) \tilde{M}^{T}\right) \xi_{i j}(t),
\end{aligned}
$$

\section{REFERENCES}

[1] M. Aldana, Boolean dynamics of networks with scale-free topology. Physica D, 185: 45-66, 2003.

[2] L. Arnold, Random Dynamical Systems. Springer-Verlag, Berlin, 1998.

[3] P. Balasubramaniam and R. Rakkiyappan, Global asymptotic stability of stochastic recurrent neural networks with multiple discrete delays. Appl. Math. Comput. doi: 10.1016/j.amc.2008.05.001 (in press).

[4] S. Boyd, L. E. Ghaoui, E. Feron and V. Balakrishnan, Linear Matrix Inequalities in System and Control Theory. Philadelphia: SIAM, 1994.

[5] C. Dangalchev, Generation models for scale-free networks. Physica A, 338: 659-671, 2004.

[6] H. Gao, J. Lam and G. Chen, New criteria for synchronization stability of general complex dynamical networks with coupling delays. Physics Letters A, 360: 263-273, 2006.

[7] H. Gao and T. Chen, Network-based $\mathcal{H}_{\infty}$ output tracking control. IEEE Trans. Auotmatic Control, 53(3): 655-667, 2008.

[8] H. Gao, P. Shi and J. Wang, Parameter-dependent robust stability of uncertain time-delay systems. J. Computational and Applied Mathematics, 206(1): 366-373.

[9] Y. He, G. P. Liu, D. Rees and M. Wu, Stability analysis for neural networks with time-varying interval delay. IEEE Transactions on Neural Networks, 18(6): 1850-1854, 2007.

[10] Khalil, H. K. Nonlinear systems. Upper Saddle River, NJ: Prentice-Hall. (1996).

[11] R.Z. Khasminskii, Stochastic Stability of Differential Equations. Alphen aan den Rijn, Sijthoffand Noor, Khasminskiidhoff, 1980.

[12] K.Q. Gu, V.L. Kharitonov, J. Chen, Stability of time-delay systems. Boston: Birkhauser, 2003.

[13] A. N. Langville and W. J. Stewart, The Kronecker product and stochastic automata networks. Journal of Computational and Applied Mathematics, 167: 429-447, 2004.

[14] C.P. Li, W.G. Sun and J. Kurths, Synchronization of complex dynamical networks with time delays. Physica A, 361: 24-34, 2006.

[15] Z. Li and G. Chen, Global synchronization and asymptotic stability of complex dynamical networks. IEEE Trans. Circuits Syst.-II, 53(1): 28-33, 2006.

[16] J. Liang, Z. Wang and X. Liu, Exponential synchronization of stochastic delayed discrete-time complex networks. Nonlinear Dynamics, 53(1-2): 153-165, 2008. 
[17] J. Liang, Z. Wang, Y. Liu and X. Liu, Global synchronization control of general delayed discrete-time networks with stochastic coupling and disturbances, IEEE Trans. Systems, Man, and Cybernetics - Part B, 38: 1073-1083, 2008.

[18] X. Liu and T. Chen, Exponential synchronization of nonlinear coupled dynamical networks with a delayed coupling. Physica A, 381: 82-92, 2007.

[19] Y. Liu, Z. Wang and X. Liu, Global exponential stability of generalized recurrent neural networks with discrete and distributed delays. Neural Networks, 19(5): 667-675, 2006.

[20] Y. Liu, Z. Wang and X. Liu, On synchronization of coupled neural networks with discrete and unbounded distributed delays. Int. J. Computer Math., 85(8): 1299-1313, 2008.

[21] W. Lu and T.Chen, Synchronization analysis of linearly coupled networks of discrete time systems. Physica D, 198: 148-168, 2004.

$[22]$ Y. Lv, W. Lv and J. Sun, Convergence dynamics of stochastic reaction-diffusion recurrent neural networks with continuously distributed delays. Nonlinear Analysis: Real World Applications, 9: 1590-1606, 2008.

[23] X. Mao, Stochastic differential equations and their applications, Horwood Publishing, 1997.

[24] X. Mao, Exponential stability of stochastic delay interval systems with markovian switching. IEEE Transanctions on automatic control, 47(10), 1604-1612, 2002.

[25] S. Mou, H. Gao, W. Qiang and K. Chen, New delay-dependent exponential stability for neural networks with time delay. IEEE Trans. Systems, Man, and Cybernetics - Part B, 38(2), 571-576, 2008.

[26] S. Mou, Y. Zhao, H. Gao and W. Qiang, Further improvement on synchronization stability of complex networks with coupling delays. Int. J. Computer Math., 85(8), 1255-1263, 2008

[27] J.E., Slotine, W. Li, Applied Nonlinear Control. Prentice-Hall, New Jersy (1991).

[28] Q. Song and Z. Wang, Stability analysis of impulsive stochastic Cohen-Grossberg neural networks with mixed time delays. Physica A: Statistical Mechanics and its Applications. 387(13): 3314-3326, 2008.

[29] Y. Sun, J. Cao and Z. Wang, Exponential synchronization of stochastic perturbed chaotic delayed neural networks. Neurocomputing, 70: 2477-2485, 2007.

[30] S. Strogatz, Exploring complex networks. Nature, 410: 268-276, 2001.

[31] Z. Toroczkai, Complex networks: the challenge of interaction topology. Los Alamos Science, 29: 94-109, 2005.

[32] X. Wang and G. Chen, Complex networks: small-world, scale-free, and beyond. IEEE Circuits Syst. Mag., 3: 6-20, 2003.

[33] Y. Wang, Z. Wang and J. Liang, A delay fractioning approach to global synchronization of delayed complex networks with stochastic disturbances. Physics Letters A 372(9): 6066-6073, 2008.

[34] Z. Wang, Y. Liu and X. Liu, $H_{\infty}$ filtering for uncertain stochastic time-delay systems with sector-bounded nonlinearities. Automatica, $44(5): 1268-1277,2008$.

[35] Z. Wang, F. Yang and X. Liu, Robust filtering for systems with stochastic nonlinearities and deterministic uncertainties, Proceedings of IMechE - Journal of Systems and Control Engineering, 220(3): 171-182., 2006.

[36] D. Williams, Probability with martingales. Cambridge University Press, Cambridge, 1991.

[37] F. Yang, Z. Wang and D. W. C. Ho, Robust mixed $H_{2} / H_{\infty}$ control for a class of nonlinear stochastic systems, IEE Proceedings - Control Theory and Applications, 153(2): 175-184, 2006.

[38] W. Yu, J. Cao and J. Lv, Global synchronization of linearly hybrid coupled networks with time-varying delay. SIAM J. Applied Dynamical Systems, 7(1): 108-133, 2008.

[39] D. Yue, E. Tian, Z. Wang and J. Lam, Stabilization of systems with probabilistic interval input delays and its applications to networked control systems, IEEE Transactions on Systems, Man and Cybernetics - Part A, in press, 2008 